\title{
Photonic Crystal Architecture for Room-Temperature Equilibrium Bose-Einstein Condensation of Exciton Polaritons
}

\author{
Jian-Hua Jiang ${ }^{1}$ and Sajeev John ${ }^{1,2}$ \\ ${ }^{1}$ Department of Physics, University of Toronto, Toronto, Ontario M5S 1A7, Canada \\ ${ }^{2}$ Department of Physics, King Abdulaziz University, Jeddah 21589, Saudi Arabia \\ (Received 9 April 2014; revised manuscript received 6 June 2014; published 13 August 2014)
}

\begin{abstract}
We describe photonic crystal microcavities with very strong light-matter interaction to realize roomtemperature, equilibrium, exciton-polariton Bose-Einstein condensation (BEC). This goal is achieved through a careful balance between strong light trapping in a photonic band gap (PBG) and large exciton density enabled by a multiple quantum-well (QW) structure with a moderate dielectric constant. This approach enables the formation of a long-lived, dense $10-\mu \mathrm{m}-1-\mathrm{cm}$ - scale cloud of exciton polaritons with vacuum Rabi splitting that is roughly $7 \%$ of the bare exciton-recombination energy. We introduce a woodpile photonic crystal made of $\mathrm{Cd}_{0.6} \mathrm{Mg}_{0.4} \mathrm{Te}$ with a 3D PBG of 9.2\% (gap-to-central-frequency ratio) that strongly focuses a planar guided optical field on CdTe QWs in the cavity. For 3-nm QWs with 5-nm barrier width, the exciton-photon coupling can be as large as $\hbar \Omega=55 \mathrm{meV}$ (i.e., a vacuum Rabi splitting of $2 \hbar \Omega=110 \mathrm{meV}$ ). The exciton-recombination energy of $1.65 \mathrm{eV}$ corresponds to an optical wavelength of $750 \mathrm{~nm}$. For $N=106$ QWs embedded in the cavity, the collective exciton-photon coupling per QW $(\hbar \Omega / \sqrt{N}=5.4 \mathrm{meV})$ is much larger than the state-of-the-art value of $3.3 \mathrm{meV}$, for the CdTe Fabry-Pérot microcavity. The maximum BEC temperature is limited by the depth of the dispersion minimum for the lower polariton branch, over which the polariton has a small effective mass of approximately $10^{-5} m_{0}$, where $m_{0}$ is the electron mass in vacuum. By detuning the bare exciton-recombination energy above the planar guided optical mode, a larger dispersion depth is achieved, enabling room-temperature BEC. The $\mathrm{BEC}$ transition temperature ranges as high as $500 \mathrm{~K}$ when the polariton density per QW is increased to $\left(11 a_{B}\right)^{-2}$, where $a_{B} \simeq 3.5 \mathrm{~nm}$ is the exciton Bohr radius and the exciton-cavity detuning is increased to $30 \mathrm{meV}$. A high-quality PBG can suppress exciton radiative decay and enhance the polariton lifetime to beyond 150 ps at room temperature, sufficient for thermal equilibrium BEC.
\end{abstract}

DOI: 10.1103/PhysRevX.4.031025

\section{INTRODUCTION}

Semiconductor microcavities offer an unique platform to study fundamental phenomena of quantum electrodynamics in the strong-coupling regime [1-3]. Thanks to advances in growth and manipulation technologies, both excitons and cavity photons are tunable in a variety of ways that enable observations of novel phenomena [3-5]. In the last decade, nonequilibrium exciton-polariton BoseEinstein condensation (BEC) has been observed in 1D Fabry-Pérot microcavities in various materials [4-9]. However, the polariton lifetime is often too short to achieve thermal equilibrium BEC. Moreover, the BEC temperature is limited by the weakness of photon confinement and the resulting insufficient exciton-photon-coupling strength.

In an optical cavity, an atomic excitation and a photon may couple together to form a coherent superposition when their decay rates (times $\hbar$ ) are much smaller than their

Published by the American Physical Society under the terms of the Creative Commons Attribution 3.0 License. Further distribution of this work must maintain attribution to the author(s) and the published article's title, journal citation, and DOI.
Subject Areas: Optics, Optoelectronics, Photonics

coupling. In a semiconductor microcavity [1-3], excitons and photons constitute polaritons in this manner. The quantum superposition of excitons and photons confers them with remarkable dynamics. For instance, the polariton effective mass, inherited from the cavity photon, is about $10^{-10}$ times the mass of a rubidium atom. Consequently, the $\mathrm{BEC}$ transition temperature of polariton gases is usually above $1 \mathrm{~K}$. In the last decade, signatures of nonequilibrium polariton BEC have been reported in semiconductor microcavities [4-9]. Although aspects of macroscopic coherence and superfluidity have been observed or claimed [6,10-13], the polariton lifetime has been too short (about $1 \mathrm{ps}$ ) to achieve genuine thermal equilibrium [14]. Recently highquality-factor Fabry-Pérot microcavities have provided longer polariton lifetimes [15]. Nevertheless, polaritons can radiatively decay into extraneous and degenerate optical modes of these $1 \mathrm{D}$ periodic structures from a micron-scale confinement region.

Because of the half-photon and half-exciton nature of the polariton, the Bose condensate contains polariton-polariton interactions (i.e., optical nonlinearity). Such a nonlinear, coherent light source has potential applications as a laser with a low excitation threshold and novel photon statistics 
[6,7,16-18]. Nonlinearity can also be exploited for the development of all-optical transistors [19], diodes [20], and switches [21,22]. Unfortunately, most realizations of polariton BEC have been at cryogenic temperatures. Above room temperature, polariton BEC [23] is essential for broad practical applications. While studies on GaN [24], $\mathrm{ZnO}$ [25,26], and organic materials [27] have found roomtemperature polariton light-emission and lasing, these polaritons are not sufficiently long lived for equilibrium BEC. These room-temperature effects are facilitated by a large exciton-binding energy $E_{b}$ and exciton-photon coupling $\hbar \Omega$. With a small exciton Bohr radius $a_{B}$, the saturation density $\simeq\left(5 a_{B}\right)^{-2}$, at which electron-hole pairs unbind due to many-body effects (screening and phasespace filling, as revealed in Ref. [28]), is likewise very high in these materials. On the other hand, small excitons are very sensitive to local disorder, which leads to strong inhomogeneous broadening that smears the exciton and polariton dispersion. The inhomogeneous broadening in the polymer material measured from optical spectroscopy in Ref. [27] is as high as $60 \mathrm{meV}$, while the exciton-photon coupling is $\hbar \Omega=58 \mathrm{meV}$. The inhomogeneous broadenings in $\mathrm{GaN}$ and $\mathrm{ZnO}$ are around $16 \mathrm{meV}$ [29] and $25 \mathrm{meV}$ [30], respectively, while the exciton-photon couplings are $25 \mathrm{meV}$ [31] and $29 \mathrm{meV}$ [32], respectively. In contrast, the inhomogeneous broadening in GaAs and $\mathrm{CdTe}$ is around $1 \mathrm{meV}$ [33]. Nevertheless, the excitonpolariton lifetime is still below 1 ps [26] in these materials due to the nature of the utilized optical cavity. Recently, the macroscopic occupation of photonic states in microcavities filled with dye molecules has been observed at room temperature [34,35]. This quasicondensate [36] in the weak-coupling regime exhibits a strongly fluctuating photon number [35], unlike BEC of polaritons in the strongcoupling regime with conserved particle number [4].

In this article, we introduce an alternative approach, using 3D photonic band-gap (PBG)-based [37,38] microcavities [39-41] to greatly enhance the exciton-photon coupling and eliminate the radiative decay of polaritons. We focus on CdTe-based photonic crystal microcavities which offer an effective balance between strong light confinement and high exciton density. The exciton-binding energy in CdTe quantum wells (QWs) has been measured to be about $20 \mathrm{meV}$ (varying with $\mathrm{QW}$ width, temperature, etc.) [42-44], leading to an exciton Bohr radius $a_{B}$ of about $4 \mathrm{~nm}$. An exciton-photon coupling of $\hbar \Omega=13 \mathrm{meV}$ has been experimentally realized in CdTe-based Fabry-Pérot microcavities with $N=16$ QWs [8]. These data indicate that neither the exciton-binding energy nor the excitonphoton coupling in CdTe structures is greater than room temperature $k_{B} T=26 \mathrm{meV}$. Bare excitons in CdTe QWs are not stable at room temperature, and the polariton BEC temperature is below room temperature in Fabry-Pérot microcavities. Indeed, nonequilibrium polariton $\mathrm{BEC}$ in a CdTe-based Fabry-Pérot microcavity is only $19 \mathrm{~K}$ [8]. On the other hand, the refractive index of CdTe is sufficient for much stronger light trapping in the form of 3D PBG. Here, we show that using a PBG architecture, the photonic field is much more strongly focused on suitably placed QWs. Consequently, the exciton-photon coupling is enhanced beyond $50 \mathrm{meV}$ (i.e., vacuum Rabi splitting beyond $100 \mathrm{meV}$ ) which stabilizes the exciton polariton and enables equilibrium BEC above room temperature.

For excitons in CdTe, multiple QWs (MQWs) with width $3 \mathrm{~nm}$ and barrier width $5 \mathrm{~nm}$, the exciton-recombination energy is $1.65 \mathrm{eV}$ (optical wavelength $750 \mathrm{~nm}$ ) and the exciton-binding energy is calculated by the effective mass approximation [45] to be $23 \mathrm{meV}$ (larger than the $9 \mathrm{meV}$ in the GaAs QW of the same width [46]). The exciton Bohr radius of $3.5 \mathrm{~nm}$ is likewise smaller than the corresponding radius in GaAs $(8.4 \mathrm{~nm})$ [46] but larger than in $\mathrm{ZnO}$ $(1.4 \mathrm{~nm})$ and $\mathrm{GaN}(2.8 \mathrm{~nm})$ [47]. These properties of CdTe lead to stronger coupling, higher saturation density, and stronger thermal stability of excitons compared to GaAs. $\mathrm{CdTe}$ is nevertheless suitable for high-quality microcavities for which growth technology is well developed $[3,43]$. CdTe has a dielectric constant of $8.5[43,48,49]$ above the threshold for PBG formation. We propose to use $\mathrm{Cd}_{0.6} \mathrm{Mg}_{0.4} \mathrm{Te}$ as the barriers between QWs as well as for the background photonic crystal. The dielectric constant of $\mathrm{Cd}_{0.6} \mathrm{Mg}_{0.4} \mathrm{Te}$ is 7.5 [50] which gives a $\mathrm{PBG}$ in the woodpile architecture (see Fig. 1) of $\delta \omega / \omega_{c}=9.2 \%$. $(\delta \omega$ denotes band-gap width, and $\omega_{c}$ is the PBG central frequency.) The dielectric constant of the regions with MQWs is taken to be 8 , which is the average value of the QW and barrier materials. Using $N=106$ QWs, the exciton-photon coupling is as large as $\hbar \Omega=55 \mathrm{meV}$; i.e., the vacuum Rabi splitting is $110 \mathrm{meV}$. The excitonphoton coupling per QW of $\hbar \Omega / \sqrt{N}=5.4 \mathrm{meV}$ is considerably larger than $13 / \sqrt{16}=3.3 \mathrm{meV}$ for the Fabry-Pérot microcavity reported in Ref. [8] (i.e., exciton-photon coupling of $13 \mathrm{meV}$ for $16 \mathrm{QWs}$ ). We also calculate the exciton-photon coupling when the number of QWs in each rod is reduced by increasing the barrier width (keeping two QWs in the central slab). The exciton-photon coupling is $45 \mathrm{meV}$ if the barrier width is increased to $10 \mathrm{~nm}$, while there are seven QWs in each rod in the active region (total of $58 \mathrm{QWs).} \mathrm{When} \mathrm{the} \mathrm{barrier} \mathrm{width} \mathrm{is}$ increased to $20 \mathrm{~nm}$ with four QWs in each rod in the active region (total of $34 \mathrm{QWs}$ ), the exciton-photon coupling is $40 \mathrm{meV}$. If there are only two QWs in the central slab and no QW in the rods, the exciton-photon coupling remains as high as $33 \mathrm{meV}$. All these energies are greater than room temperature $k_{B} T=26 \mathrm{meV}$ and (with positive detuning of the exciton-recombination energy from the lowest 2D guided mode) are shown below to support room-temperature polariton BEC.

Fabrication of the structure proposed [as shown in Figs. 1(a) and 1(b)] is possible using wafer fusion and the laser-beam-assisted precise alignment of rods [51,52]. 


\section{(a)}
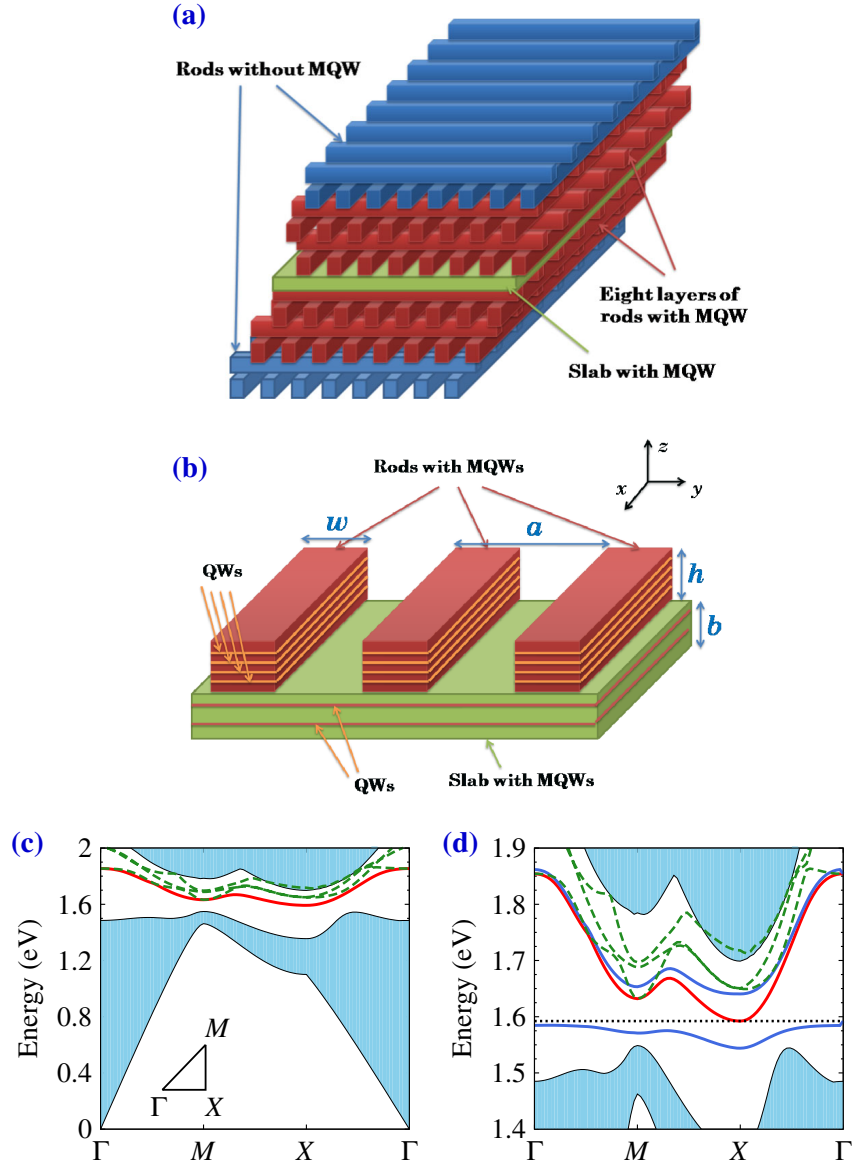

FIG. 1. Photonic crystal architecture and photonic band dispersion. (a) A slab layer sandwiched between two woodpile photonic crystals induces $2 \mathrm{D}$ photonic bands ("guided modes") in the 3D PBG with $\delta \omega / \omega_{c}=9.2 \%$, where $\delta \omega$ is the band-gap width and $\omega_{c}$ is the PBG central frequency. (b) QWs are grown in the slab and the eight nearby layers of rods. Typically, $a \simeq 360 \mathrm{~nm}$, $w=0.25 a \simeq 90 \mathrm{~nm}, \quad h=0.3 a \simeq 110 \mathrm{~nm}, \quad$ and $\quad b=0.06 a \simeq$ $20 \mathrm{~nm}$. There are about $100 \mathrm{QWs}$ with the same width. There are two QWs in the slab layer and about $12 \mathrm{QWs}$ in each rod. The QW widths are chosen to be 3, 4, 5, and $6 \mathrm{~nm}$ for comparison, while the width of the barrier layers between QWs is fixed to be $5 \mathrm{~nm}$. QWs are made of CdTe (dielectric constant 8.5), while the barriers and photonic crystals are made of $\mathrm{Cd}_{0.6} \mathrm{Mg}_{0.4} \mathrm{Te}$ (dielectric constant 7.5). In the regions with QWs, the dielectric constant is taken to be 8 (average of the two materials). (c),(d) Dispersion of the 2D guided modes (dashed green and solid red curves) and the bulk 3D photonic bands (shaded regions). The first Brillouin zone of the $2 \mathrm{D}$ guided modes is $-\frac{\pi}{a} \leq q_{x}, q_{y}<\frac{\pi}{a}$. The lowest 2D photonic band [the solid curves in (c) and (d)] is coupled with excitons [dotted curve in (d)] in QWs. The lower and upper polariton branches [solid blue curves in (d)] are depicted for exciton recombination in resonance with the lowest $2 \mathrm{D}$ guided mode. In this case, each QW width is $4 \mathrm{~nm}$, the excitonrecombination energy is $E_{X 0}=1.59 \mathrm{eV}$, and the photonic lattice constant is chosen to be $a=360 \mathrm{~nm}$. The exciton-photoncoupling strength and the on-resonance lower-polariton dispersion depth is $48 \mathrm{meV}$. Larger dispersion depth and higher BEC temperature are achieved by detuning the exciton-recombination energy above the lowest 2D guided mode.
It has been demonstrated [40] that a layer of rods with MQWs (grown by molecular beam epitaxy and etched) can be placed in a woodpile photonic crystal. By repeated wafer fusion, laser-assisted alignment, careful etching, and polishing (monitored by thin-film interference), sandwich structures can be fabricated with high precision. Accurate fabrication of GaAs woodpile photonic crystals with a near-infrared, MQW, light-emitting layer was reported by Noda and co-workers [40]. Similar techniques can, in principle, be employed for our proposed CdTe photonic crystal structure.

3D photonic crystal microcavities have been fabricated with much higher quality factors than their counterpart Fabry-Pérot microcavities. Quality factors as high as $2 \times 10^{6}$ have been demonstrated experimentally for microcavities in the woodpile architecture [53]. In addition, a 3D PBG eliminates the purely radiative recombination of excitons into unwanted modes when the lower polariton energy is in the PBG. In this case, the polariton lifetime is determined by exciton nonradiative recombination. In CdTe QWs at room temperature, the excitonphotoluminescence-decay time is measured to be $150 \mathrm{ps}$ in Ref. [54], suggesting that the nonradiative recombination time is even longer. This time scale is sufficient for polaritons to reach thermal equilibrium since the excitonoptical-phonon scattering in CdTe at room temperature is quite efficient [55]. Our calculation reveals that roomtemperature equilibrium polariton $\mathrm{BEC}$ can be achieved for a polariton density of $5 \times 10^{3} \mu \mathrm{m}^{-2}$ [exciton density in each QW of approximately $\left(30 a_{B}\right)^{-2}$, below the saturation density of $\left(5 a_{B}\right)^{-2}$, where $a_{B}=3.5 \mathrm{~nm}$ is the bare exciton Bohr radius for a $\mathrm{QW}$ of width $3 \mathrm{~nm}$ ]. This condition occurs when the exciton-recombination energy is detuned $30 \mathrm{meV}$ above the lowest 2D guided mode and the excitons are trapped in a box with a side length of $10-10^{4} \mu \mathrm{m}$. The realization of equilibrium polariton $\mathrm{BEC}$ at and above room temperature may open the door to broad applications of quantum mechanics beyond the microscopic scale.

\section{POLARITONS IN A 3D PHOTONIC BAND-GAP MICROCAVITY}

Figures 1(a) and 1(b) depict our proposed woodpile photonic crystal heterostructure. The distance between the nearest-neighbor rods in the same layer is denoted by $a$, the height of each rod is $h=0.3 a$, the width of each rod is $w=0.25 a$, and the thickness of the solid central slab is $b=0.06 a$. In order to place the lowest $2 \mathrm{D}$ guided mode within the PBG in resonance with the exciton transition at $1.59 \mathrm{eV}$ (for QW width $4 \mathrm{~nm}$ ), typically, $a=360 \mathrm{~nm}$. The PBG is centered in the range of $750 \mathrm{~nm}$ [56]. MQWs are grown in the slab and the eight layers of rods close to the slab. The other regions above and below are the cladding woodpile photonic crystals. The QWs are made of CdTe, while the barrier layers between QWs are made of $\mathrm{Cd}_{0.6} \mathrm{Mg}_{0.4} \mathrm{Te}$. Both materials are direct electronic band-gap semiconductors with band 
structures similar to GaAs. The electronic band gaps of CdTe and $\mathrm{Cd}_{0.6} \mathrm{Mg}_{0.4}$ Te are 1.475 and $2.28 \mathrm{eV}$, respectively [57,58] (from linear interpolation between the band gaps of CdTe and $\mathrm{MgTe}$, the latter being $3.49 \mathrm{eV}$ ). The cladding rods without QWs are also made of $\mathrm{Cd}_{0.6} \mathrm{Mg}_{0.4} \mathrm{Te}$. The structure breaks the translation symmetry along the $z$ direction [see Fig. 1(a)] but has lattice translation symmetry in the $x-y$ plane. [The coordinate axes are depicted in Fig. 1(b).] There is no inversion symmetry with respect to the slab. However, the system has a $D_{2 d}$ symmetry with two mirror planes: the $y-z$ and $x-z$ planes [59]. There are several 2D guided photonic bands localized around the slab. The dispersion of those confined modes within the PBG are shown in Figs. 1(c) and 1(d). The electric field in these 2D bands can be written as

$$
\overrightarrow{\mathcal{E}}_{i, \vec{q}}(\vec{r})=\sqrt{\frac{\hbar \omega_{i, \vec{q}}}{2 \varepsilon_{0} S}} \vec{u}_{i, \vec{q}}(\vec{r}) e^{i \vec{q} \cdot \vec{\rho}} .
$$

Here, $\varepsilon_{0}$ is the vacuum permittivity, $S$ is the area of the structure in the $x-y$ plane, $\vec{r}=(\vec{\rho}, z)$ with $\vec{\rho}=(x, y)$, and $\omega_{i, \vec{q}}$ is the frequency of a photon in the $i$ th band with a Bloch wave vector $\vec{q}$ in the $2 \mathrm{D}$ Brillouin zone $-\frac{\pi}{a} \leq q_{x}, q_{y}<\frac{\pi}{a}$. The field is normalized such that $S^{-1} \int d \vec{\rho} d z \varepsilon(\vec{r})\left|\vec{u}_{i, \vec{q}}(\vec{r})\right|^{2}=1$, where $\varepsilon(\vec{r})$ is the coordinatedependent dielectric function. Photons in the lowest confined photonic band and electron-heavy-hole excitons in the MQWs interact to form the lower and upper polariton branches. These dispersions are depicted in Fig. 1(d) when the exciton-recombination energy coincides with the 2D photonic band edge. The dispersion of the lowest confined photonic band and that of the lower polariton branch in the 2D photonic Brillouin zone are shown in Fig. 2 for the case when the exciton-recombination energy is detuned by $30 \mathrm{meV}$ above the 2D photonic band edge.

The energy levels and wave functions of the $1 s$ excitonic states in QWs are obtained from diagonalizing the effective
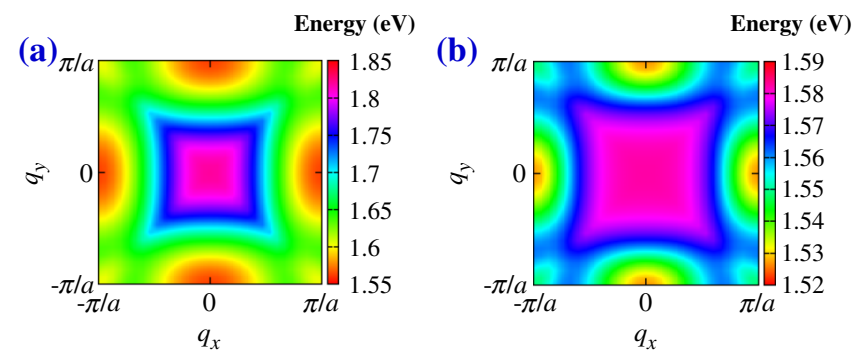

FIG. 2. Dispersion of confined photonic and polaritonic bands in 2D wave-vector space. (a) Dispersion of the lowest confined photonic band in the first Brillouin zone $-\frac{\pi}{a} \leq q_{x}, q_{y}<\frac{\pi}{a}$. The lattice constant is $a=365 \mathrm{~nm}$. (b) Dispersion of the lower polariton branch in the same wave-vector region. The $\mathrm{QW}$ width is $4 \mathrm{~nm}$. The lower band edge $(1.56 \mathrm{eV})$ of guided $2 \mathrm{D}$ modes within the $3 \mathrm{D}$ PBG is $30 \mathrm{meV}$ below the exciton-recombination energy $\left(E_{X 0}=1.59 \mathrm{eV}\right)$. The exciton-photon coupling of $48 \mathrm{meV}$ involves the collective response of $100 \mathrm{QWs}$. mass Hamiltonian [45]. (See the Appendix for details.) Combining the microscopic details of cavity photons and QW excitons, the Hamiltonian of the coupled excitonphoton system [46] is given by

$$
\begin{aligned}
H & =H_{X}+H_{P}+H_{\mathrm{int}}, \\
H_{X} & =\sum_{l, \alpha, n, \vec{q}} E_{X}^{(l)}\left(\vec{q}+\vec{G}_{n}\right) \beta_{l, \alpha, \vec{q}+\vec{G}_{n}}^{\dagger} \beta_{l, \alpha, \vec{q}+\vec{G}_{n}}, \\
H_{P} & =\sum_{i, \vec{q}} \hbar \omega_{i, \vec{q}} a_{i, \vec{q}}^{\dagger} a_{i, \vec{q}}, \\
H_{\text {int }} & =\sum_{l, \alpha, n, i, \vec{q}} i \hbar \bar{\Omega}_{l, \alpha, n, i, \vec{q}} \beta_{l, \alpha, \vec{q}+\vec{G}_{n}}^{\dagger} a_{i, \vec{q}}+\text { H.c. }
\end{aligned}
$$

The operator $\beta_{l, \alpha, \vec{q}+\vec{G}_{n}}^{\dagger}$ creates a $1 s$ exciton in the $l$ th QW with polarization $\alpha=L, T$ (longitudinal or transverse) and center-of-mass wave vector $\vec{q}+\vec{G}_{n}$, where $\vec{G}_{n}=\frac{2 \pi}{a}\left(n_{x}, n_{y}\right)$ is the $2 \mathrm{D}$ reciprocal lattice vector for the photonic band structure with integers $n_{x}$ and $n_{y}$. The exciton dispersion $E_{X}^{(l)}(\vec{q})=E_{X}(\vec{q})+\delta E_{l}$ with $E_{X}(\vec{q}) \equiv E_{X 0}+\left(\hbar^{2} q^{2} / 2 m_{X}\right)$ includes a fluctuation term $\delta E_{l}$ varying for different QWs to describe disorder effects. $E_{X 0}$ is the exciton-recombination energy at zero center-of-mass wave vector. $m_{X}=m_{e}+m_{h}$ is the effective mass of an exciton, with $m_{e}$ and $m_{h}$ being the electron and hole effective masses, respectively. The operator $a_{i, \vec{q}}^{\dagger}$ creates a photon in the $i$ th band with Bloch wave vector $\vec{q}$. The coupling-matrix element is calculated [46] as

$$
\begin{aligned}
\bar{\Omega}_{l, \alpha, n, i, \vec{q}}= & \frac{|\phi(0)| d \sqrt{\omega_{i, \vec{q}}}}{S_{\mathrm{UC}} \sqrt{2 \hbar \varepsilon_{0}}} \\
& \times \int_{\mathrm{UC}} d \vec{\rho} e^{-i \overrightarrow{G_{n}} \cdot \vec{\rho}} u_{\alpha, i, \vec{q}}\left(\vec{\rho}, z_{l}\right) \Theta\left(\vec{\rho}, z_{l}\right) .
\end{aligned}
$$

$|\phi(0)|$ denotes the amplitude of the 1 s exciton wave function when the distance between electron and hole is $0 . d$ is the interband dipole-matrix element in CdTe. $z_{l}$ is the coordinate of the center of the $l$ th $\mathrm{QW}$ in the $z$ direction. $S_{\mathrm{UC}}=a^{2}$ is the area of the unit cell (UC) in the $x-y$ plane. $u_{\alpha, i, \vec{q}}=\overrightarrow{\mathbf{e}}_{\alpha} \cdot \vec{u}_{i, \vec{q}}$, where $\overrightarrow{\mathbf{e}}_{\alpha}$ is a unit vector along the polarization direction of the $\alpha$ exciton. For longitudinal ( $\alpha=L$ ) excitons, $\overrightarrow{\mathbf{e}}_{\alpha}$ is along $\vec{q}$, while for the transverse $(\alpha=T)$ exciton, it is perpendicular to both $\vec{q}$ and the $z$ direction. $\Theta(\vec{r})$ equals 1 when the coordinate $\vec{r}$ is in the semiconductor region and 0 when $\vec{r}$ is in the air region. $\Theta(\vec{r})$ describes the overlap between the photonic field and the excitonic wave function. The integration in Eq. (3) is over the UC of the structure in the $x-y$ plane.

It is sufficient to use only the spectrum close to the energy minima of the lowest confined photonic band to calculate the dispersion of the lower polariton branch [see Figs. 1(d) and 2(b)]. This branch is essential for the study of 
polariton BEC. There are two energy minima for the photon: one at $\vec{Q}^{(x)}=\left(\frac{\pi}{a}, 0\right)$, the other at $\vec{Q}^{(y)}=\left(0, \frac{\pi}{a}\right)$. We consider the situation when the exciton-recombination energy $E_{X 0}$ is close to the two energy minima. The photonic dispersion around the two minima is approximately parabolic:

$$
\hbar \omega_{\vec{q}}=\hbar \omega_{0}+\frac{\hbar^{2}\left(q_{x}-Q_{x}^{(\nu)}\right)^{2}}{2 m_{x}^{(\nu)}}+\frac{\hbar^{2}\left(q_{y}-Q_{y}^{(\nu)}\right)^{2}}{2 m_{y}^{(\nu)}},
$$

with $\nu=x, y$. From the $D_{2 d}$ symmetry, the photonic effective masses satisfy the relations $m_{x}^{(x)}=m_{y}^{(y)}$ and $m_{x}^{(y)}=m_{y}^{(x)}$. If $a=360 \mathrm{~nm}$, the plane-wave-expansion calculation [60] yields $m_{x}^{(x)}=2.9 \times 10^{-5} m_{0}$ and $m_{x}^{(y)}=$ $4.7 \times 10^{-5} m_{0}$, where $m_{0}$ is the electron mass in vacuum. Scale invariance of the Maxwell equations dictates that $\omega_{0}, m_{x}^{(x)}$, and $m_{y}^{(x)}$ are proportional to $1 / a$. For CdTe QWs, the effective mass of the conduction-band electron is $m_{e}=0.095 m_{0}$ [58], while the effective mass of the heavy hole is $m_{h}=0.4 m_{0}$ [48].

When the fluctuation in the exciton energy among QWs is neglected, i.e., $E_{X}^{(l)}(\vec{q})=E_{X}(\vec{q})$ for all $l$, we introduce the collective excitonic operator $b_{\vec{q}} \equiv \sum_{l, \alpha, n} \frac{\Omega_{l, \alpha, n, \vec{q}}}{\Omega_{\vec{q}}} \beta_{l, \alpha, n, \vec{q}}$ to obtain the effective polariton Hamiltonian $[61,62]$ :

$$
\begin{aligned}
H & =H_{0}+H_{I}, \\
H_{0} & =\sum_{\vec{q}}\left[E_{X}(\vec{q}) b_{\vec{q}}^{\dagger} b_{\vec{q}}+\hbar \omega_{\vec{q}} a_{\vec{q}}^{\dagger} a_{\vec{q}}\right], \\
H_{I} & =\sum_{\vec{q}} i \hbar \Omega_{\vec{q}}\left(b_{\vec{q}}^{\dagger} a_{\vec{q}}-a_{\vec{q}}^{\dagger} b_{\vec{q}}\right) .
\end{aligned}
$$

The collective exciton-photon coupling is given by

$$
\hbar \Omega_{\vec{q}}=\hbar \sqrt{\sum_{l, \alpha, n}\left|\bar{\Omega}_{l, \alpha, n, \vec{q}}\right|^{2}} .
$$

Here, $b_{\vec{q}}$ corresponds to the mode that couples most strongly to the lowest 2D guided mode in the PBG. Other excitonic modes orthogonal to this mode (in the Hilbert space of excitonic states spanning all QWs) are much more weakly coupled to the $2 \mathrm{D}$ photonic bands. In the above equations, the photonic band index $i$ is omitted since only the lowest confined $2 \mathrm{D}$ photonic band is relevant. We also approximate the $\vec{q}$ dependence of the collective coupling by its value at the two polariton-energy minima and define $\hbar \Omega \equiv \hbar \Omega_{\vec{Q}^{(x)}}=\hbar \Omega_{\vec{Q}^{(y)}}$. This approximation is justified since beyond the narrow range around the two energy minima, the lower polariton dispersion becomes excitonlike and its detailed form does not alter the low-energy dynamics [see Figs. 1(d) and 2(b)]. The dispersion of the lower polariton branch is given by

$$
\begin{aligned}
E_{\mathrm{LP}}(\vec{q})= & \frac{E_{X}(\vec{q})+\hbar \omega_{\vec{q}}}{2} \\
& -\left[\left(\frac{E_{X}(\vec{q})-\hbar \omega_{\vec{q}}}{2}\right)^{2}+\hbar^{2} \Omega^{2}\right]^{1 / 2} .
\end{aligned}
$$

The above dispersion can likewise be extended to the whole 2D wave-vector space since away from the energy minima $\vec{Q}^{(x)}$ and $\vec{Q}^{(y)}$, the dispersion of the lower polariton becomes excitonlike (i.e., almost flat dispersion).

\section{VERY STRONG LIGHT-MATTER INTERACTION}

Figure 3(a) shows the collective exciton-photon coupling $\hbar \Omega$ calculated for different $\mathrm{QW}$ widths when excitons are in resonance with the lowest $2 \mathrm{D}$ guided mode for photons, i.e., $E_{X 0}=\hbar \omega_{0}$. The vacuum Rabi splitting $2 \hbar \Omega$ exceeds $100 \mathrm{meV}$ when the QW width is $3 \mathrm{~nm}$ (experimentally accessible, as shown by Refs. [63,64]). The width of the barrier layers between QWs is fixed at $5 \mathrm{~nm}$ throughout this work. Figure 3(b) depicts how the photonic crystal lattice constant $a$ must be adjusted as the QW width is varied, in order to maintain resonance between the excitonrecombination energy and the $2 \mathrm{D}$ guided mode. The total number of QWs that fit into the active region is correspondingly adjusted. Figure 3(c) depicts the variation of the exciton-binding energy and Bohr radius with QW width. The calculated exciton-binding energies, ranging from 17 to $23 \mathrm{meV}$, agree well with the experimental results of 17-25 meV [42-44].

Considerable enhancement of the exciton-photon coupling in our photonic crystal microcavity (compared with Fabry-Pérot microcavities) originates from the light-trapping effect of the PBG. In Fig. 3(d), we compare the photonic field-intensity $|\overrightarrow{\mathcal{E}}|^{2}$ distribution as a function of $z$ but averaged over the $x-y$ plane for the $\mathrm{Cd}_{0.4} \mathrm{Mg}_{0.6} \mathrm{Te}-\mathrm{Cd}_{0.8} \mathrm{Mn}_{0.2} \mathrm{Te}$ Fabry-Pérot and the $3 \mathrm{D}$ photonic crystal microcavities with the same photon energy. The photonic crystal microcavity clearly focuses the electric field much more strongly than the Fabry-Pérot. Moreover, the averaged electric field intensity at $z=0$ is significantly larger. This large field intensity is because in photonic crystal, a large volume fraction $(\simeq 75 \%)$ is air, which reduces the overall dielectric constant of the structure. The reduced screening enhances the averaged electric field intensity $|\overrightarrow{\mathcal{E}}|^{2}$ at $z=0$ by 16 times. Another important advantage of the PBG heterostructure is the strong spatial variations of the field intensity in the $x-y$ plane. The higherintensity regions act as an attractive "optical potential" for the exciton, and the wave function of the lower polariton is peaked in these "hot spots" which offers a way to "pattern" the polariton condensates in the $x-y$ plane [see Fig. 3(e)]. The peak intensity in the $x-y$ plane is greater than the average intensity depicted in Fig. 3(d) which provides further enhancement in exciton-photon coupling compared 

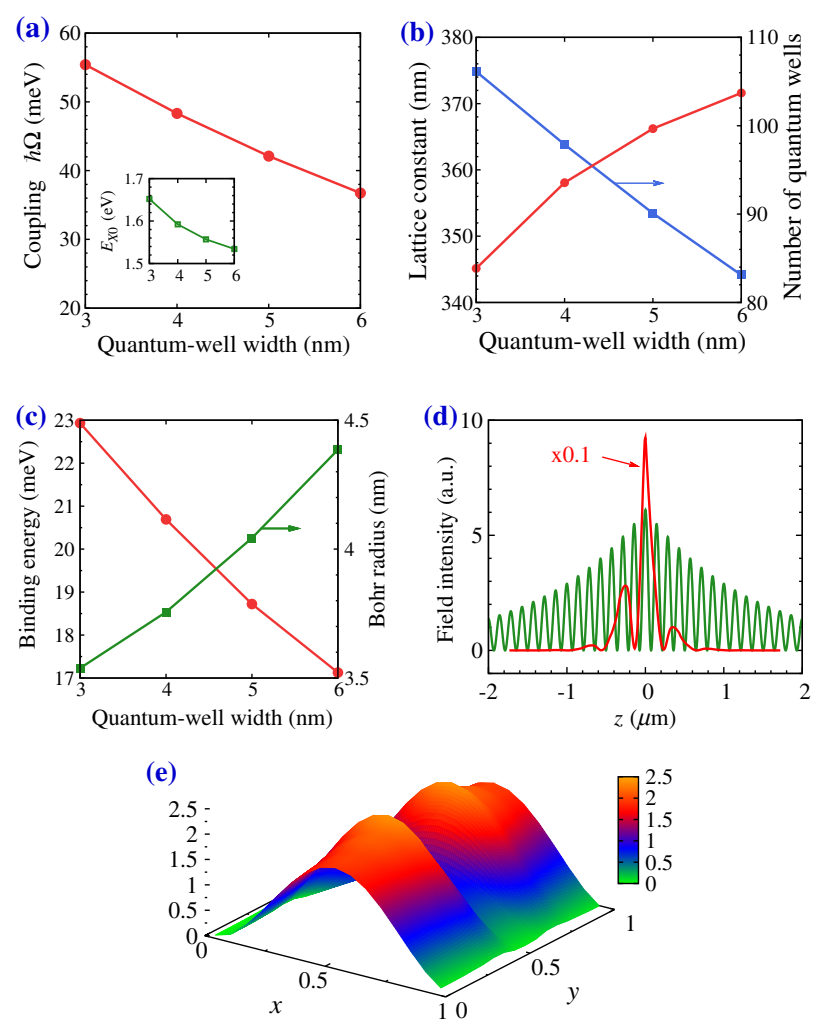

FIG. 3. Very strong light-matter interaction in the photonic band-gap 2D guided mode. (a) Exciton-photon collective interaction strength $\hbar \Omega$ as a function of QW width for a fixed barrier width of $5 \mathrm{~nm}$. The horizontal axis spans from $N=106$ to 83 QWs. Inset: Exciton-recombination energy $E_{X 0}$. The lowest 2D photonic band edge $\hbar \omega_{0}$ is kept in resonance with the excitonrecombination energy $E_{X 0}$ as the $\mathrm{QW}$ width is varied. The barrier layers between QWs are made of $\mathrm{Cd}_{0.6} \mathrm{Mg}_{0.4} \mathrm{Te}$. (b) As $E_{X 0}$ varies with the $\mathrm{QW}$ width, the photonic crystal lattice constant $a$ is adjusted (red curve) to keep $\hbar \omega_{0}=E_{X 0}$. Since $a$ changes, the total number $N$ of QWs (blue curve) in the slab and rods also changes. (c) Exciton-binding energy and Bohr radius for different QW widths calculated using an effective mass approximation [45] (see the Appendix). (d) Average electric field-intensity $S^{-1} \int d \vec{\rho}|\overrightarrow{\mathcal{E}}(\vec{\rho}, z)|^{2}$ profile at the 2D guided mode edge in the PBG cavity (red curve) compared to that in the $\mathrm{Cd}_{0.4} \mathrm{Mg}_{0.6} \mathrm{Te}^{-\mathrm{Cd}_{0.8}} \mathrm{Mn}_{0.2} \mathrm{Te}$ Fabry-Pérot cavity [44] (green curve) along the $z$ direction. The photons in both cavities have a band-edge energy of $1.59 \mathrm{eV}$. The photonic field intensity in the PBG cavity is multiplied by a factor of 0.1 in the figure. The Fabry-Pérot cavity is a $\lambda / 2$ cavity with $\lambda=780 \mathrm{~nm}$. The lattice constant of the photonic crystal is $a=360 \mathrm{~nm}$. (e) Fieldintensity $|\overrightarrow{\mathcal{E}}|^{2}$ profile in the $x-y$ plane (in one unit cell of photonic crystal) at $z=0$. Here, the averaged intensity is 1 . The $x$ and $y$ coordinates are in units of the photonic lattice constant $a$.

to the 1D Fabry-Pérot geometry for which the field intensity is uniform in the $x-y$ plane. From Figs. 3(d) and 3(e), the peak intensity is about 38 times (the average intensity is 16 times) as large as that in the Fabry-Pérot microcavity. Other photonic crystals that have a larger PBG, such as the slanted-pore photonic crystals [65], provide even stronger light focusing and larger excitonphoton couplings than we have presented here using the woodpile architecture.

Another advantage of PBG light trapping is that the quality factor of the microcavity can be very high. A quality factor of $2 \times 10^{6}$ with nanosecond photonic lifetime has been experimentally demonstrated in the woodpile architecture [53]. This quality factor can be further enhanced by simply increasing the thickness of the cladding photonic crystal. Our calculation indicates that moderate fabrication imperfection does not reduce the quality factor considerably due to the existence of the 3D PBG. (See the end of Sec. IV) When radiative decay is strongly inhibited, polaritons can decay through slower nonradiative processes. The Auger recombination rate [66] is estimated as about $10^{-3} \mathrm{~s}$ (see the Appendix). Nonradiative recombination triggered by electronic impurity states, such as the Shockley-Read-Hall mechanism [67], may be more important in polariton decay. Nevertheless, the measured photoluminescence-decay time of $150 \mathrm{ps}$ in a CdTe QW at room temperature [54] suggests that the nonradiative polariton-decay time should be longer than 150 ps. The nonradiative exciton-decay time is believed to be on the order of nanoseconds [4]. Clearly, very strong excitonphoton coupling, long polariton lifetime, and small polariton effective mass are possible in the photonic crystal microcavity.

\section{ROOM-TEMPERATURE POLARITON BEC}

In this section, we delineate the precise conditions for room-temperature polariton $\mathrm{BEC}$ in our photonic crystal microcavity. The BEC transition temperature is very sensitive to the dispersion depth of the lower polariton branch and the density of polaritons, but relatively insensitive to the polariton-trapping area over a broad range of lengths $D$, from $10 \mu \mathrm{m}$ to $1 \mathrm{~cm}$, when the polariton density is high. The polariton density can be controlled by excitation of electron-hole pairs with energy above the upper 3D photonic band edge. These carriers can then relax into the lower polariton branch within the PBG. Since the polariton lifetime is enhanced beyond the thermalization time (on the order of ps at room temperature), polaritons achieve thermal equilibrium long before they decay. We consider a finite-sized trap with box (square-well potential) confinement in the $x-y$ plane. This square quantum box has a side length of $D$. Such a box can be created by replacing our infinite central slab by a finite-sized slab $[40,53,68]$ or by increasing the $\mathrm{Mg}$ fraction outside the prescribed box region [69] to create a barrier for excitons. Trapping by these means enables a higher BEC transition temperature than harmonic trapping potentials induced by strain [18]. The trapping box induces quantization of the polariton wave vector in the $x$ and $y$ directions, discretizing the polariton spectra and enabling the BEC phase transition that would otherwise be forbidden in an infinite 2D system. 
To simplify the calculation, we approximate the polariton effective mass with the isotropic "density-of-states mass" $m_{\mathrm{DOS}}=\sqrt{m_{x}^{(x)} m_{x}^{(y)}}$.

In Figs. 4(a) and 4(b), we plot the ground-state occupation $N_{0}$ as a function of the polariton density. The polariton distribution is calculated assuming a noninteracting, equilibrium polariton gas. There is a rapid increase of $N_{0}$ around a threshold density, above which $N_{0}$ becomes comparable with the total polariton number $N_{\text {tot }}$. We define the polariton BEC transition according to the criterion $N_{0} / N_{\text {tot }}=0.1$ [70]. This criterion gives a lower estimate of the BEC transition temperature than the criterion employed by Ketterle and van Druten [71]. At low temperatures, polaritons are mainly distributed in the low-energy, photonlike states around the bottom of the lower polariton branch with very small effective mass [see Fig. 4(c)]. The small effective mass enhances condensation and reduces the threshold density for polariton BEC. The dispersion depth of these photonlike states is

$$
\left.V \equiv\left(E_{X}-E_{\mathrm{LP}}\right)\right|_{\vec{Q}^{(\nu)}}=\frac{\Delta}{2}+\sqrt{\left(\frac{\Delta}{2}\right)^{2}+\hbar^{2} \Omega^{2}},
$$

where $\Delta=E_{X 0}-\hbar \omega_{0}$ is the detuning between the exciton and the photon. On the other hand, if the temperature is comparable to or higher than $V / k_{B}$, the occupation of highenergy, excitonlike states is significant. The effective mass of the bare exciton is around 4 orders of magnitude larger than the 2D guided photon effective mass. Likewise, the density of excitonlike states is about 4 orders of magnitude larger than the low-energy, photonlike states. The consequences are indicated in Figs. 4(a) (the low-temperature case) and 4(b) (the high-temperature case). Polariton BEC at temperatures comparable with $V / k_{B}$ require very high polariton density. Since the polariton is a quantum superposition of a photon and an exciton, the polariton density cannot exceed a specific "saturation density" beyond which exciton-exciton interaction causes considerable exciton ionization. Therefore, the polariton BEC transition temperature is essentially limited by the dispersion depth $V$. The dispersion depth is increased by a positive detuning $\Delta$ [see Fig. 4(d)]. However, the excitonic fraction of the polariton is reduced with increasing $\Delta$, which reduces the phonon-polariton and polariton-polariton interactions, and consequently, it takes a longer time for polaritons to reach their thermal equilibrium. From experimental measurements [55], the phonon-exciton-scattering time at room temperature is estimated to be about $0.1 \mathrm{ps}$ (see the Appendix). A thermalization time on the order of $1 \mathrm{ps}$ is possible if the exciton fraction is higher than $10 \%$. In the range of detuning considered in this article $0 \mathrm{meV}<\Delta<40 \mathrm{meV}$, the excitonic fraction is greater than $19 \%$ for exciton-photon coupling as low as $\hbar \Omega=25 \mathrm{meV}$. Both the excitonic fraction and the
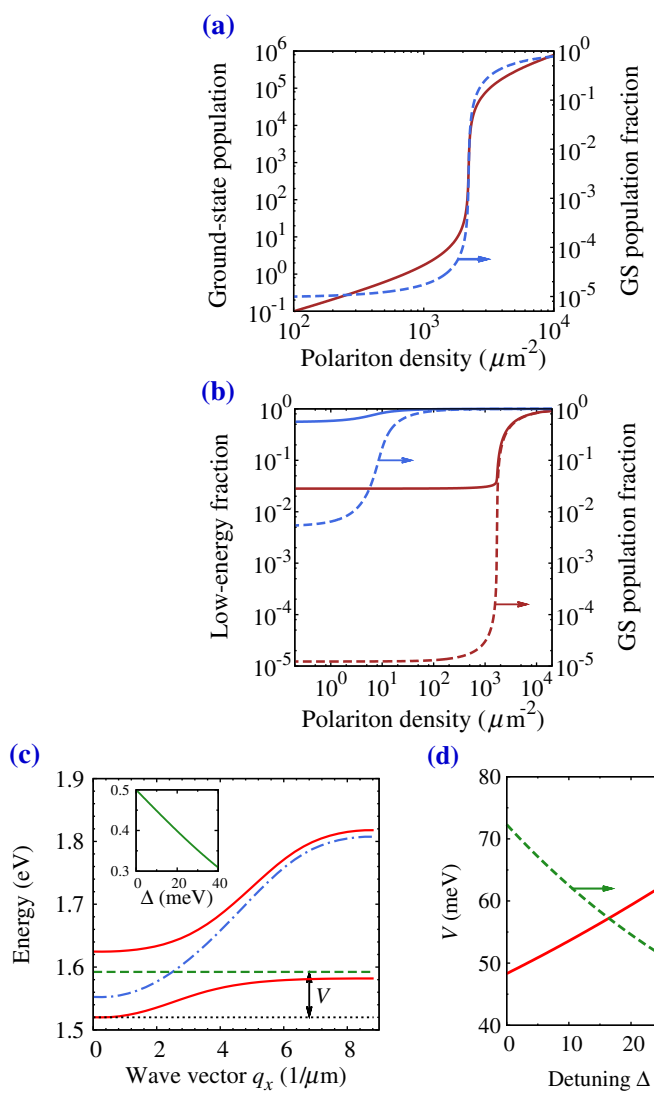

(d)

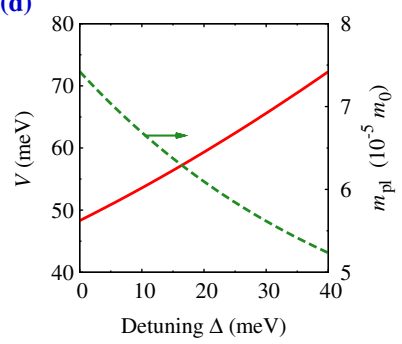

FIG. 4. Polariton population on the ground state and other low-energy states for the exciton-box trap with $D=10 \mu \mathrm{m}$, an exciton-photon coupling of $\hbar \Omega=48 \mathrm{meV}$, and an excitonrecombination energy of $E_{X 0}=1.59 \mathrm{eV}$. The QW width is $4 \mathrm{~nm}$, and the barrier-layer width is $5 \mathrm{~nm}$. The detuning is (a) $\Delta=30 \mathrm{meV}$ and (b) $\Delta=40 \mathrm{meV}$. (a) Population of the ground state (GS) vs polariton density (solid curve). The rapid, nonlinear increase of the GS population indicates the BEC transition. The GS population fraction (dashed curve), i.e., the GS population divided by the total population, is of order unity for densities beyond the BEC transition. (b) GS population fraction (dashed curves) and the population fraction on the low-energy discrete states (solid curves) below the continuum exciton-recombination energy $E_{X 0}$. (There are more than 1000 such states.) These low-energy states are photonlike states with very small effective mass, whereas states higher than $E_{X 0}$ are excitonlike. When a large population fraction resides in the photonlike states, the BEC threshold density is reduced. For $T=100 \mathrm{~K}$ (blue curves), populated polariton states are mostly photonlike. The threshold density is much lower than for $T=300 \mathrm{~K}$ (brown curves), where the population fraction on low-energy states is negligible. (c) The dispersion of polaritons (solid red curves), bare excitons (dashed green curve), and pure photons (dash-dotted blue curve) for detuning $\Delta=40 \mathrm{meV}$ with $q_{y}=\pi / a=8.6 \mu \mathrm{m}^{-1}$ for $\mathrm{a}=370 \mathrm{~nm}$. The dispersion depth of the lower polariton branch $V$ is also depicted. (The dotted black line denotes the lower polariton band edge.) Inset: Excitonic fraction of the lower polariton branch as a function of detuning $\Delta$. (d) The dispersion depth $V$ (solid red curve) and polariton effective mass $m_{\mathrm{pl}}$ (dashed green curve) of the lower polariton branch vs the detuning $\Delta$. 
effective mass of the lower-polariton dispersion minimum decrease with detuning $\Delta$, as shown in the inset of Fig. 4(c) and in Fig. 4(d), respectively.

We calculate the polariton BEC transition temperature $T_{c}$ for various $\mathrm{QW}$ widths, exciton-trap sizes, detunings, and exciton-photon couplings for a fixed polariton density. The results are plotted in Fig. 5. Clearly, room-temperature polariton BEC is attainable at overall polariton density $\gtrsim 3 \times 10^{3} \mu \mathrm{m}^{-2}$ which is well below the saturation density in each QW of $\left(5 a_{B}\right)^{-2}$, where $a_{B}$ is the exciton Bohr radius. The transition temperature is higher for smaller QW width since a larger number of QWs can be used. It is likewise higher for larger exciton-photon coupling and positive detuning which is consistent with the picture that $T_{c}$ is limited by the dispersion depth $V$. For large trap sizes $D \gg 10 \mu \mathrm{m}, T_{c}$ decreases as expected from the MerminWagner theorem [72] that forbids finite-temperature BEC in an infinite 2D system. However, this decrease is very slow and little degradation in $T_{c}$ is seen over the range of $10 \mu \mathrm{m}<D<1 \mathrm{~cm}$. Interestingly, the exciton-trap-size dependence of $T_{c}$ for smaller $D$ is nonmonotonic: $T_{c}$ has a peak value around a box size $D \simeq 5 \mu \mathrm{m}$. When the exciton-trap size is sufficiently small, the quantization
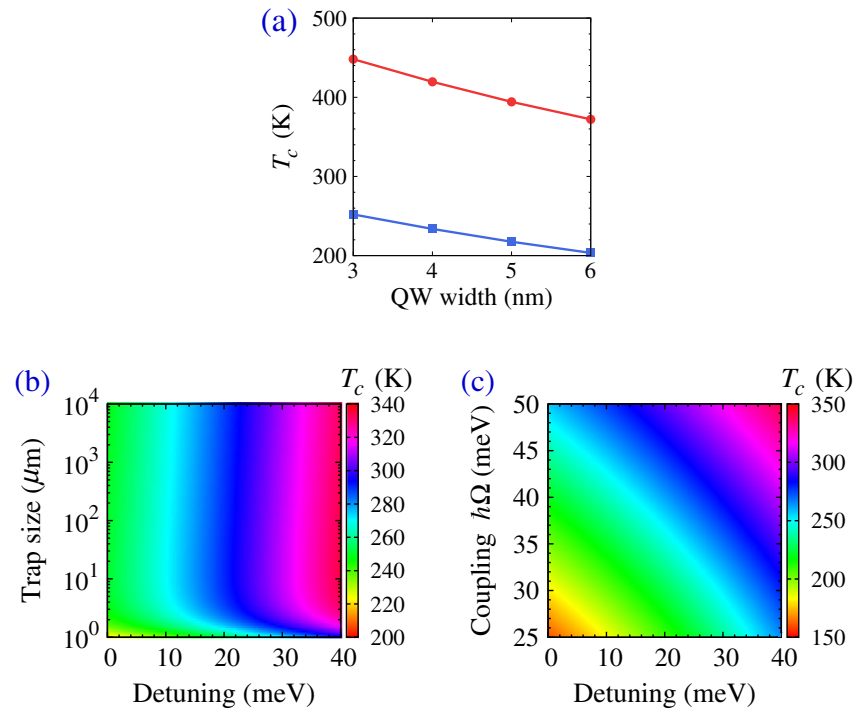

FIG. 5. Polariton BEC transition temperature $T_{c}$ at fixed densities. (a) Transition temperature $T_{c}$ with detuning $\Delta=30 \mathrm{meV}$ for different QW widths (with QWs filling an entire active region) at fixed polariton densities: $1.5 \times 10^{4} \mu \mathrm{m}^{-2}$ (curve with solid circles) and $1.9 \times 10^{3} \mu \mathrm{m}^{-2}$ (curve with solid squares). For the higher total density, the exciton density in each QW is below $\left(9 a_{B}\right)^{-2}$. For the lower total density, the exciton density in each QW is below $\left(26 a_{B}\right)^{-2}$. (b) Dependence of the transition temperature $T_{c}$ on the detuning $\Delta$ and the exciton-trap size $D$ when the exciton-photon coupling is $\hbar \Omega=48 \mathrm{meV}$ (QW width $4 \mathrm{~nm}$ and barrier width $5 \mathrm{~nm}$ ) and the polariton density is $3 \times 10^{3} \mu \mathrm{m}^{-2}$. (c) Dependence of the transition temperature $T_{c}$ on the detuning $\Delta$ and the exciton-photon coupling $\hbar \Omega$ when the exciton-trap size is $D=10 \mu \mathrm{m}$ and the polariton density is $3 \times 10^{3} \mu \mathrm{m}^{-2}$. energy of the cavity (e.g., the energy difference between the first excited state and the ground state) can be comparable to or larger than the dispersion depth $V$. The condensation then crosses over from polaritonlike to excitonlike. Since the exciton effective mass is much larger than that of the polariton, the transition temperature is rapidly reduced.

The polariton density used in our calculation is experimentally accessible. For MQWs, the total exciton density is distributed among the QWs and the excitonic fraction in each QW of a polariton may be much less than unity. For example, using $N=100 \mathrm{QWs}$, each of width $4 \mathrm{~nm}$ and barrier width $5 \mathrm{~nm}$ (i.e., $\hbar \omega_{0}=1.56 \mathrm{eV}$ and $E_{X 0}=$ $1.59 \mathrm{eV}$ with $a=365 \mathrm{~nm}$ and $a_{B}=3.8 \mathrm{~nm}$ ), the collective exciton-photon coupling is $48 \mathrm{meV}$. For a detuning of $\Delta=30 \mathrm{meV}$, the largest excitonic fraction in any single $\mathrm{QW}$ is 5.5\%. [The exciton fraction varies among QWs since the exciton-photon coupling for each QW is different; see Eq. (3)] For total polariton density $1.5 \times 10^{4} \mu \mathrm{m}^{-2}$, the exciton density in each QW is no larger than $\left(9 a_{B}\right)^{-2}$, where $a_{B}$ is the exciton Bohr radius. This single QW exciton density is less than the saturation density $[28,44] \simeq\left(5 a_{B}\right)^{-2}$.
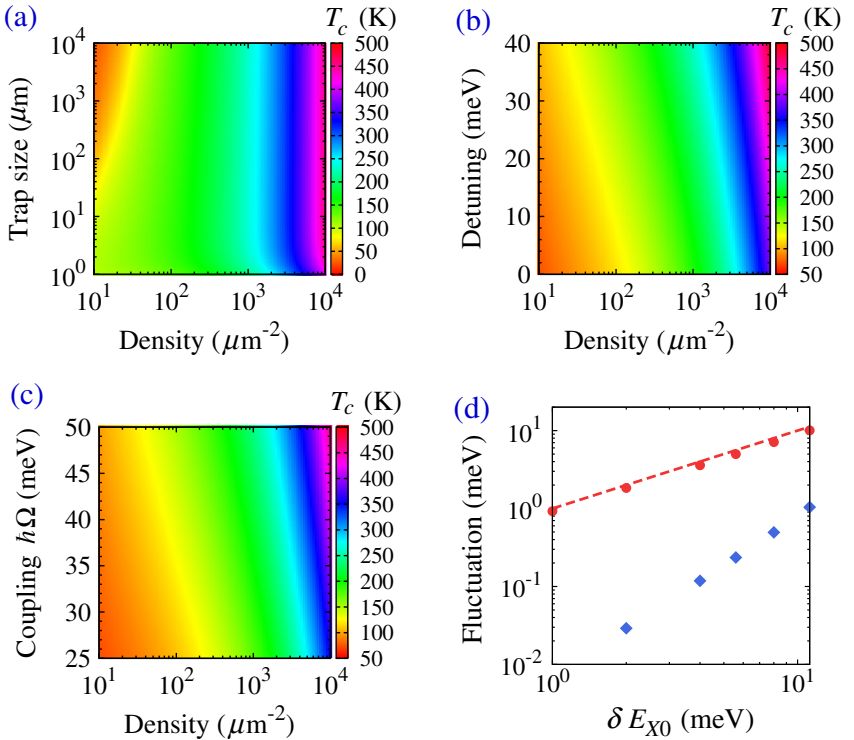

FIG. 6. BEC transition temperature $T_{c}$ as a function of total polariton density and (a) box-trap size, (b) exciton-photon detuning, and (c) exciton-photon-coupling strength. (d) Rootmean-square deviation of the vacuum Rabi splitting (diamonds) and lower-polariton dispersion depth (circles) as a function of exciton inhomogeneous broadening $\delta E_{X 0}$. In (a) and (b), the exciton-photon coupling is $\hbar \Omega=48 \mathrm{meV}$ (QW width $4 \mathrm{~nm}$ and barrier width $5 \mathrm{~nm}$ ). For (a) and (c), the detuning is $30 \mathrm{meV}$. For (b) and (c), the exciton-box-trap size is $10 \mu \mathrm{m}$. The excitonrecombination energy is $E_{X 0}=1.59 \mathrm{eV}$. For (d), the detuning is $\Delta=0$. The dashed curve in (d) depicts the value of $\delta E_{X 0}$ itself for reference. 
Systematic dependence of the BEC transition temperature on the total polariton density is shown in Fig. 6. Figure 6(a) reveals that the exciton-trap-size dependence of $T_{c}$ is much more pronounced at low polariton density, where the transition temperature is much smaller than $V / k_{B}$. Here, most polaritons are low-energy photonlike states with very small effective mass. The transition temperature at fixed density is limited mainly by the level spacing between the ground and first excited states [18,71], which depends sensitively on the exciton-trap size. In contrast, at high polariton density, the transition temperature is close to $V / k_{B}$. When the dispersion depth $V$ is the main factor limiting $T_{c}$, the exciton-trap size has marginal influence. Figures 6(b) and 6(c) reveal that large detuning and/or exciton-photon coupling is favorable for roomtemperature polariton BEC because both of them enhance the dispersion depth $V$ [see Fig. 4(d)].

Finally, we discuss the effect of fabrication disorder which can randomize sensitive parameters such as the detuning $\Delta$ and the collective exciton-photon coupling $\hbar \Omega$. We show that the former effect is more significant. Photonic disorder arises from fluctuation of the shape and size of the rods and slabs constituting the photonic crystal. Electronic disorder arises from impurities, dislocations, (local) QW width fluctuation, random strains, etc. In addition to these forms of inhomogeneous broadening, dynamic fluctuations (homogeneous broadening) arise from phonon-polariton and polariton-polariton interactions which are particularly important at high temperatures.

Plane-wave-expansion calculation indicates that the 2D photonic band edge varies by less than $2 \%$ of its value if the fabrication error is smaller than $20 \mathrm{~nm}$ for woodpile rods outside the active region, less than $12 \mathrm{~nm}$ for rods in the active woodpile region, and less than $6 \mathrm{~nm}$ for the central slab thickness. The effect of electronic disorder has been studied earlier [62] for the single QW case, where it was found that polaritons remain robust due to their small effective mass and highly mobile half-photon nature. Electronic scale disorder is largely averaged out in a scenario similar to motional narrowing [62].

For the MQW structures studied here, there are also fluctuations of exciton energy among QWs due to variations in QW width or lateral confinement of the rods. This broadening is modeled by adding a random energy $\delta E_{l}$ to the exciton dispersion in the $l$ th $\mathrm{QW} E_{X}^{(l)}(\vec{q})=E_{X 0}+$ $\frac{\hbar^{2} q^{2}}{2 m_{X}}+\delta E_{l} . \delta E_{l}$ is modeled as a Gaussian random variable with variance $\left(\delta E_{X 0}\right)^{2}$. The effects of this disorder on vacuum Rabi splitting $2 \hbar \Omega$ and polariton-dispersion depth $V$ are presented in Fig. 6(d). The calculation is performed by averaging over $10^{5}$ random configurations. The standard deviation of the vacuum Rabi splitting is very small due to the collective nature of the polariton. The fluctuation in polariton energy consists of the sum of random excitonenergy variations from each of the $N$ QWs. The total effect is an average effect that has a much smaller variance, according to the central limit theorem [46] $\frac{\delta \Omega}{\Omega} \sim \mathcal{O}(1 / \sqrt{N})$.

The fluctuation of the polariton-dispersion depth $V$ is approximately the same as the inhomogeneous broadening of the exciton $\delta E_{X 0}$. The randomization of the detuning $\Delta$ directly affects this dispersion depth [see Eq. (8)]. This effect is much more significant than the fluctuation of the vacuum Rabi splitting $2 \hbar \Omega$. Experimental measurements in a single CdTe-CdMgTe QW give a typical inhomogeneous broadening of $1 \mathrm{meV}$ [33]. Hence, intra-QW inhomogeneous broadening can be rendered small with presentday fabrication capabilities. Inter-QW inhomogeneous broadening estimated from the lateral confinement difference for QWs in rods and in the central slab is less than $1 \mathrm{meV}$ as well.

Finally, dynamic fluctuations (such as polariton-phonon and polariton-polariton scattering) cause both homogeneous broadening and the relaxation of polaritons. The latter is crucial for polaritons to reach the ground state and achieve thermal equilibrium BEC. However, homogeneous broadening reduces the polariton-dispersion depth $V$, and consequently, $T_{c}$. In CdTe QWs, at room temperature, experiments [55] suggest a homogeneous linewidth of $\Gamma=22 \mathrm{meV}$ (see the Appendix). The broadening of the cavity photon is negligible for high-quality-factor $\left(2 \times 10^{6}\right.$ as shown in Ref. [53]) photonic crystal microcavities. If we estimate the effect of homogeneous broadening by adding an imaginary part $i \Gamma$ to the exciton energy, the vacuum Rabi splitting at zero detuning $(\Delta=0)$ is reduced to $2 \sqrt{\hbar^{2} \Omega^{2}-\Gamma^{2} / 4}$ [73]. More importantly, the dispersion depth of the lower polariton for nonzero detuning becomes

$$
V=\frac{\Delta}{2}+\operatorname{Re} \sqrt{\left(\frac{\Delta-i \Gamma}{2}\right)^{2}+\hbar^{2} \Omega^{2}} .
$$

In general, exciton-phonon interaction reduces the dispersion depth $V$. However, for $\Delta=30 \mathrm{meV}, \hbar \Omega=$ $48 \mathrm{meV}$, and $\Gamma=22 \mathrm{meV}$, the lower-polariton dispersion depth is reduced by only $1.1 \mathrm{meV}$ which is equivalent to a reduction of detuning by $2 \mathrm{meV}$ and has negligible effects on the transition temperature, according to Fig. 6(b). These results suggest that our very strongly coupled excitonphoton system can withstand considerable homogeneous and inhomogeneous broadening and still deliver equilibrium room-temperature polariton $\mathrm{BEC}$.

\section{CONCLUSION}

In summary, we have identified a CdTe-based photonic band-gap architecture with a range of different quantum-well embeddings that are predicted to support room-temperature (or higher) equilibrium Bose-Einstein condensation of exciton polaritons. The most crucial factors in enabling high-temperature BEC are the dispersion depth of the lower polariton branch and the 
total areal density of long-lived excitons within the trapping region. The dispersion depth is determined by the collective exciton-photon-coupling strength and is enhanced by detuning the bare exciton-recombination energy above the lower edge of a slow-light, 2D guided photonic band within the larger 3D photonic band gap. The 3D PBG enables considerably stronger light trapping than conventional 1D Fabry-Pérot microcavities, leading to stronger exciton-photon coupling. The collective exciton-phononcoupling strength is further enhanced by embedding up to about 100 quantum wells in the photonic crystal regions adjacent to a central slab region containing two principal quantum wells. Positive detuning of the excitonrecombination energy relative to the strongly coupled photon mode is made possible by the 3D PBG. The positively detuned exciton remains within the 3D PBG and it cannot decay radiatively into extraneous optical modes that leave the system.

The total areal density of excitons is enhanced by distributing them over a large number of quantum wells that are all strongly coupled to the 2D guided mode within the 3D PBG. The use of CdTe-based architectures offers a suitable balance between photonic and electronic properties. The relatively low dielectric constant of CdTe compared to other popular materials (such as GaAs) enables a small exciton Bohr radius and higher saturation exciton density. Nevertheless, the dielectric constant is sufficiently large enough to facilitate a 3D PBG that encompasses the lower-polariton dispersion depth and provides strong $3 \mathrm{D}$ light confinement.

Our calculations of the BEC transition temperature are based on a noninteracting polariton gas confined in a $2 \mathrm{D}$ box trap. Previous studies [18] have shown that a slight reduction in the BEC transition, together with polariton antibunching effects, are expected when exciton-exciton interactions are included in the microscopic Hamiltonian. While a box trap of 5- $\mu \mathrm{m}$ side length is predicted to yield the highest BEC transition temperature, very little degradation of $T_{c}$ is seen for box dimensions up to $1 \mathrm{~cm}$ when polariton density is high. This result suggests that BEC may be observable in a finitesize photonic crystal up to centimeter-scale lateral dimension, without recourse to a separately engineered 2D trap.

It is significant that for total polariton densities approaching $10^{4} \mu \mathrm{m}^{-2}$, the exciton density per quantum well remains below $\left(10 a_{B}\right)^{-2}$, where $a_{B}$ is the exciton Bohr radius. At these densities, our PBG architecture provides sufficiently strong exciton-photon coupling and polaritondispersion depth to provide a predicted BEC transition as high as $500 \mathrm{~K}$, in the absence of additional homogeneous and inhomogeneous broadening effects. This architecture offers a safe margin for the realization of room-temperature $\mathrm{BEC}$ in realistic systems with both foreseen and unforeseen fabrication imperfections, exciton-phonon-scattering processes, and other damping effects. In this way, the photonic band-gap architecture provides a realistic prospect of bringing the magic of macroscopic quantum mechanics to the realm of everyday experience.

\section{ACKNOWLEDGMENTS}

This work was supported by the Natural Sciences and Engineering Research Council of Canada, the Canadian Institute for Advanced Research, and the United States Department of Energy Contract No. DE-FG02-10ER46754.

\section{APPENDIX: CALCULATION METHODS AND DETAILS}

The spectrum and wave function of the photonic bands are calculated by solving the Maxwell equations using the plane-wave-expansion method [60]. In calculating the photonic bands in the $\mathrm{Cd}_{0.4} \mathrm{Mg}_{0.6} \mathrm{Te}-\mathrm{Cd}_{0.8} \mathrm{Mn}_{0.2}$ Te FabryPérot cavity, the dielectric constant for $\mathrm{Cd}_{0.4} \mathrm{Mg}_{0.6}$ Te is 7.0 (linear interpolation between the dielectric constant of CdTe is 8.5 [49], and that of $\mathrm{MgTe}$ is 6.0 [50]), while the dielectric constant of $\mathrm{Cd}_{0.8} \mathrm{Mn}_{0.2} \mathrm{Te}$ is 7.8 [74]. The electronic structures are calculated using the effective mass approximation [45]. The effective mass of the electron is $m_{e}=0.095 m_{0}$, with $m_{0}$ being the bare electron mass in vacuum [58]. For the [001]-grown QWs concerned in this work, the heavy-hole effective mass along the growth direction is $0.6 m_{0}$ [75], while the effective mass in the QW plane is $m_{h}=0.4 m_{0}$ [48]. The energy and wave functions of the electron and hole subbands are calculated using a finite-depth well model [45]. The electronic band gap of CdTe is $E_{g}=1.475 \mathrm{eV}[58,76]$, while the electronic band gap for MgTe is $3.49 \mathrm{eV}$ [77]. Linear interpolation yields the band gap of $\mathrm{Cd}_{0.6} \mathrm{Mg}_{0.4} \mathrm{Te}$ as $2.28 \mathrm{eV}$. The bandgap difference between $\mathrm{Cd}_{0.6} \mathrm{Mg}_{0.4} \mathrm{Te}$ and CdTe is $0.8 \mathrm{eV}$. The offset for the conduction band between those two materials is $70 \%$ of their band-gap difference [78]. The interband dipole-matrix element is given by

$$
d=\frac{e \hbar \sqrt{E_{\mathrm{CV}}}}{E_{g} \sqrt{2 m_{0}}},
$$

where $E_{\mathrm{CV}}=2 P_{\mathrm{CV}}^{2} / m_{0}$ is the energy related to the momentum-matrix element $P_{\mathrm{CV}}$ between the conduction and valence bands. From Ref. [78], we take $E_{\mathrm{CV}}=21 \mathrm{eV}$. Material parameters in the above are taken for room temperature.

The exciton spectrum and wave functions are calculated by diagonalizing the exciton Hamiltonian. For our narrow QWs, we consider only the excitons formed by electrons and holes in their lowest subband [43]. We ignore the mixing with higher subbands and numerically diagonalize the exciton Hamiltonian within the lowest subband. This calculation yields an exciton-binding energy comparable to the experimentally observed one. The exciton wave amplitude $|\phi(0)|$ and exciton Bohr radius are also obtained, using the same considerations. 
The Auger recombination time $\tau_{A}$ is estimated using the method presented in Ref. [66]. For a volume exciton density of $10^{17} \mathrm{~cm}^{-3}$ (volume density of excitons corresponding to an areal polariton density of $10^{4} \mu \mathrm{m}^{-2}$, estimated by dividing the areal polariton density by the thickness of the active region) in CdTe, $\tau_{A} \simeq 10^{-3} \mathrm{~s}$. This very small Auger recombination rate is due to the large band gap and small carrier density. Another nonradiative decay mechanism is the Shockley-Read-Hall mechanism due to electronic trapping centers within the semiconductor band gap. However, experiments in high-quality CdTe QWs have shown a photoluminescence-decay time of $150 \mathrm{ps}$ at room temperature [54] which places a lower bound on the nonradiative decay time.

The polariton-thermalization time is estimated as follows. Experiments [55] have found that exciton homogeneous broadening at high temperature is dominated by interactions with longitudinal-optical (LO) phonons. The exciton linewidth is given by $A_{\mathrm{LO}} /\left[\exp \left(E_{\mathrm{LO}} / k_{B} T\right)-1\right]$, where $E_{\mathrm{LO}}=21.3 \mathrm{meV}$ is the LO phonon energy [58] and the parameter $A_{\mathrm{LO}}=15 \mathrm{meV}$ is fitted from the measured temperature dependence of the exciton linewidth in Ref. [55]. Using this result, we estimate that the exciton linewidth due to LO phonon scattering at $T=300 \mathrm{~K}$ is $22 \mathrm{meV}$. This result suggests a polariton linewidth at zero detuning $(\Delta=0)$ of $11 \mathrm{meV}$ (the exciton fraction of the polariton is 50\%) and corresponds to a subpicosecond thermalization time. For positive detuning, if the exciton fraction in the polariton remains larger than $10 \%$, then the thermalization time is below or around $1 \mathrm{ps}$. This timescale is considerably smaller than the nonradiative decay time of the polariton and suggests that the polariton gas has sufficient time to reach thermal equilibrium.

[1] C. Weisbuch, M. Nishioka, A. Ishikawa, and Y. Arakawa, Observation of the Coupled Exciton-Photon Mode Splitting in a Semiconductor Quantum Microcavity, Phys. Rev. Lett. 69, 3314 (1992).

[2] G. Khitrova, H. M. Gibbs, M. Kira, S. W. Koch, and A. Scherer, Vacuum Rabi Splitting in Semiconductors, Nat. Phys. 2, 81 (2006).

[3] The Physics of Semiconductor Microcavities, edited by B. Deveaud (Wiley-VCH, Weinheim, 2007).

[4] H. Deng, H. Haug, and Y. Yamamoto, Exciton-Polariton Bose-Einstein Condensation, Rev. Mod. Phys. 82, 1489 (2010).

[5] I. Carusotto and C. Ciuti, Quantum Fluids of Light, Rev. Mod. Phys. 85, 299 (2013).

[6] H. Deng, G. Weihs, C. Santori, J. Bloch, and Y. Yamamoto, Condensation of Semiconductor Microcavity Exciton Polaritons, Science 298, 199 (2002).

[7] H. Deng, G. Weihs, D. Snoke, J. Bloch, and Y. Yamamoto, Polariton Lasing vs. Photon Lasing in a Semiconductor
Microcavity, Proc. Natl. Acad. Sci. U.S.A. 100, 15318 (2003).

[8] J. Kasprzak, M. Richard, S. Kundermann, A. Baas, P. Jeambrun, J. M. J. Keeling, F. M. Marchetti, M. H. Szymaska, R. André, J. L. Staehli, V. Savona, P. B. Littlewood, B. Deveaud, and L.S. Dang, Bose-Einstein Condensation of Exciton Polaritons, Nature (London) 443, 409 (2006).

[9] R. Balili, V. Hartwell, D. Snoke, L. Pfeiffer, and K. West, Bose-Einstein Condensation of Microcavity Polaritons in a Trap, Science 316, 1007 (2007).

[10] A. Amo, J. Lefrère, S. Pigeon, C. Adrados, C. Ciuti, I. Carusotto, R. Houdré, E. Giacobino, and A. Bramati, Superfluidity of Polaritons in Semiconductor Microcavities, Nat. Phys. 5, 805 (2009).

[11] K. G. Lagoudakis, M. Wouters, M. Richard, A. Baas, I. Carusotto, R. André, L. S. Dang, and B. Deveaud-Plédran, Quantized Vortices in an Excitonpolariton Condensate, Nat. Phys. 4, 706 (2008); D. Sanvitto, F. M. Marchetti, M. H. Szymańska, G. Tosi, M. Baudisch, F. P. Laussy, D. N. Krizhanovskii, M. S. Skolnick, L. Marrucci, A. Lemaítre, J. Bloch, C. Tejedor, and L. Viña, Persistent Currents and Quantized Vortices in a Polariton Superfluid, Nat. Phys. 6, 527 (2010); K. G. Lagoudakis, T. Ostatnický, A. V. Kavokin, Y. G. Rubo, R. André, and B. Deveaud-Plédran, Observation of Half-Quantum Vortices in an ExcitonPolariton Condensate, Science 326, 974 (2009).

[12] C. W. Lai, N. Y. Kim, S. Utsunomiya, G. Roumpos, H. Deng, M. D. Fraser, T. Byrnes, P. Recher, N. Kumada, T. Fujisawa, and Y. Yamamoto, Coherent Zero-State and $\pi$-State in an Exciton-Polariton Condensate Array, Nature (London) 450, 529 (2007).

[13] A. Amo, S. Pigeon, D. Sanvitto, V. G. Sala, R. Hivet, I. Carusotto, F. Pisanello, G. Leménager, R. Houdré, E. Giacobino, C. Ciuti, and A. Bramati, Polariton Superfluids Reveal Quantum Hydrodynamic Solitons, Science 332, 1167 (2011).

[14] L. V. Butov and A. V. Kavokin, The Behaviour of ExcitonPolaritons, Nat. Photonics 6, 2 (2012); B. Deveaud-Plédran, The Behaviour of Exciton-Polaritons, Nat. Photonics 6, 205 (2012).

[15] B. Nelsen, G. Liu, M. Steger, D. W. Snoke, R. Balili, K. West, and L. Pfeiffer, Dissipationless Flow and Sharp Threshold of a Polariton Condensate with Long Lifetime, Phys. Rev. X 3, 041015 (2013); M. Steger, G. Liu, B. Nelsen, C. Gautham, D. W. Snoke, R. Balili, L. Pfeiffer, and K. West, Long-Range Ballistic Motion and Coherent Flow of Long-Lifetime Polaritons, Phys. Rev. B 88, 235314 (2013).

[16] A. Imamoğlu, R. J. Ram, S. Pau, and Y. Yamamoto, Nonequilibrium Condensates and Lasers without Inversion: Exciton-Polariton Lasers, Phys. Rev. A 53, 4250 (1996).

[17] C. Schneider, A. Rahimi-Iman, N. Y. Kim, J. Fischer, I. G. Savenko, M. Amthor, M. Lermer, A. Wolf, L. Worschech, V. D. Kulakovskii, I. A. Shelykh, M. Kamp, S. Reitzenstein, A. Forchel, Y. Yamamoto, and S. Höfling, An Electrically Pumped Polariton Laser, Nature (London) 497, 348 (2013); P. Bhattacharya, B. Xiao, A. Das, S. Bhowmick, and J. Heo, 
Solid State Electrically Injected Exciton-Polariton Laser, Phys. Rev. Lett. 110, 206403 (2013).

[18] S. J. Yang and S. John, Coherence and Antibunching in a Trapped Interacting Bose-Einstein Condensate, Phys. Rev. B 84, 024515 (2011).

[19] D. Ballarini, M. De Giorgi, E. Cancellieri, R. Houdré, E. Giacobino, R. Cingolani, A. Bramati, G. Gigli, and D. Sanvitto, All-Optical Polariton Transistor, Nat. Commun. 4, 1778 (2013).

[20] H. S. Nguyen, D. Vishnevsky, C. Sturm, D. Tanese, D. Solnyshkov, E. Galopin, A. Lemaître, I. Sagnes, A. Amo, G. Malpuech, and J. Bloch, Realization of a Double-Barrier Resonant Tunneling Diode for Cavity Polaritons, Phys. Rev. Lett. 110, 236601 (2013).

[21] S. John and T. Quang, Collective Switching and Inversion without Fluctuation of Two-Level Atoms in Confined Photonic Systems, Phys. Rev. Lett. 78, 1888 (1997).

[22] A. Amo, T. C. H. Liew, C. Adrados, R. Houdré, E. Giacobino, A. V. Kavokin, and A. Bramati, ExcitonPolariton Spin Switches, Nat. Photonics 4, 361 (2010); R. Cerna, Y. Léger, T. K. Paraïso, M. Wouters, F. MorierGenoud, M. T. Portella-Oberli, and B. Deveaud, Ultrafast Tristable Spin Memory of a Coherent Polariton Gas, Nat. Commun. 4, 2008 (2013).

[23] A. Kavokin, Polaritons: The Rise of the Bosonic Laser, Nat. Photonics 7, 591 (2013); D. Snoke, Microcavity Polaritons: A New Type of Light Switch, Nat. Nanotechnol. 8, 393 (2013).

[24] S. Christopoulos, G. Baldassarri, H. von Högersthal, A. J. D. Grundy, P. G. Lagoudakis, A. V. Kavokin, J. J. Baumberg, G. Christmann, R. Butté, E. Feltin, J.-F. Carlin, and N. Grandjean, Room-Temperature Polariton Lasing in Semiconductor Microcavities, Phys. Rev. Lett. 98, 126405 (2007); Gabriel Christmann, R. Butté, Eric Feltin, J.-F. Carlin, and Nicolas Grandjean, Room Temperature Polariton Lasing in a GaN/AlGaN Multiple Quantum Well Microcavity, Appl. Phys. Lett. 93, 051102 (2008).

[25] Ying-Yu Lai, Yu-Pin Lan, and Tien-Chang Lu, Strong LightMatter Interaction in ZnO Microcavities, Light Sci. Appl. 2, e76 (2013).

[26] F. Li et al., From Excitonic to Photonic Polariton Condensate in a ZnO-Based Microcavity, Phys. Rev. Lett. 110, 196406 (2013).

[27] D. G. Lidzey, D. D. C. Bradley, T. Virgili, A. Armitage, M. S. Skolnick, and S. Walker, Room Temperature Polariton Emission from Strongly Coupled Organic Semiconductor Microcavities, Phys. Rev. Lett. 82, 3316 (1999); S. Kéna-Cohen and S. R. Forrest, Room-Temperature Polariton Lasing in an Organic Single-Crystal Microcavity, Nat. Photonics 4, 371 (2010).

[28] S. Schmitt-Rink, D. S. Chemla, and D. A. B. Miller, Theory of Transient Excitonic Optical Nonlinearities in Semiconductor Quantum-Well Structures, Phys. Rev. B 32, 6601 (1985).

[29] R. Butté, G. Christmann, E. Feltin, J.-F. Carlin, M. Mosca, M. Ilegems, and N. Grandjean, Room-Temperature Polariton Luminescence from a Bulk GaN Microcavity, Phys. Rev. B 73, 033315 (2006).

[30] R. Schmidt-Grund, B. Rheinländer, C. Czekalla, G. Benndorf, H. Hochmuth, M. Lorenz, and M. Grundmann,
Exciton-Polariton Formation at Room Temperature in a Planar ZnO Resonator Structure, Appl. Phys. B 93, 331 (2008).

[31] G. Christmann, R. Butté, E. Feltin, A. Mouti, P. A. Stadelmann, A. Castiglia, J.-F. Carlin, and N. Grandjean, Large Vacuum Rabi Splitting in a Multiple Quantum Well GaN-Based Microcavity in the Strong-Coupling Regime, Phys. Rev. B 77, 085310 (2008).

[32] J.-R. Chen, T.-C. Lu, Y.-C. Wu, S.-C. Lin, W.-R. Liu, W.-F. Hsieh, C.-C. Kuo, and C.-C. Lee, Large Vacuum Rabi Splitting in ZnO-Based Hybrid Microcavities Observed at Room Temperature, Appl. Phys. Lett. 94, 061103 (2009).

[33] G. V. Astakhov, V. A. Kosobukin, V. P. Kochereshko, D. R. Yakovlev, W. Ossau, G. Landwehr, T. Wojtowicz, G. Karczewski, and J. Kossut, Inhomogeneous Broadening of Exciton Lines in Magneto-optical Reflection from CdTel CdMgTe Quantum Wells, Eur. Phys. J. B 24, 7 (2001).

[34] J. Klaers, J. Schmitt, F. Vewinger, and M. Weitz, BoseEinstein Condensation of Photons in an Optical Microcavity, Nature (London) 468, 545 (2010).

[35] J. Schmitt, T. Damm, D. Dung, F. Vewinger, J. Klaers, and M. Weitz, Observation of Grand-Canonical Number Statistics in a Photon Bose-Einstein Condensate, Phys. Rev. Lett. 112, 030401 (2014); J. Klaers, J. Schmitt, T. Damm, F. Vewinger, and M. Weitz, Bose-Einstein Condensation of Paraxial Light, Appl. Phys. B 105, 17 (2011).

[36] D. W. Snoke, in Exciton Polaritons in Microcavities, edited by V. Timofeev and D. Sanvitto, Springer Series in Solid State Sciences Vol. 172 (Springer, New York, 2012).

[37] S. John, Strong Localization of Photons in Certain Disordered Dielectric Superlattices, Phys. Rev. Lett. 58, 2486 (1987).

[38] E. Yablonovitch, Inhibited Spontaneous Emission in SolidState Physics and Electronics, Phys. Rev. Lett. 58, 2059 (1987).

[39] O. Painter, R. K. Lee, A. Scherer, A. Yariv, J. D. O’Brien, P. D. Dapkus, and I. Kim, Two-Dimensional Photonic Band-Gap Defect Mode Laser, Science 284, 1819 (1999).

[40] S. Ogawa, M. Imada, S. Yoshimoto, M. Okano, and S. Noda, Control of Light Emission by 3D Photonic Crystals, Science 305, 227 (2004); S. Noda, M. Fujita, and T. Asano, Spontaneous-Emission Control by Photonic Crystals and Nanocavities, Nat. Photonics 1, 449 (2007).

[41] K. Hennessy, A. Badolato, M. Winger, D. Gerace, M. Atatüre, S. Gulde, S. Fält, E. L. Hu, and A. Imamoğlu, Quantum Nature of a Strongly Coupled Single Quantum Dot-Cavity System, Nature (London) 445, 896 (2007).

[42] S. R. Jackson, J. E. Nicholls, W. E. Hagston, P. Harrison, T. Stirner, J. H. C. Hogg, B. Lunn, and D. E. Ashenford, Magneto-optical Study of Excitonic Binding Energies, Band Offsets, and the Role of Interface Potentials in CdTe $/ \mathrm{Cd}_{1-x} \mathrm{Mn}_{x}$ Te Multiple Quantum Wells, Phys. Rev. B 50, 5392 (1994).

[43] R. André, D. Heger, L. S. Dang, and Y. M. d'Aubigné, Spectroscopy of Polaritons in CdTe-Based Microcavities, J. Cryst. Growth 184-185, 758 (1998).

[44] L. S. Dang, D. Heger, R. André, F. Bœuf, and R. Romestain, Stimulation of Polariton Photoluminescence in Semiconductor Microcavity, Phys. Rev. Lett. 81, 3920 (1998). 
[45] P. Harrison, Quantum Wells, Wires and Dots (Wiley, New York, 2001).

[46] J.-H. Jiang and S. John (to be published).

[47] S. Faure, T. Guillet, P. Lefebvre, T. Bretagnon, and B. Gil, Comparison of Strong Coupling Regimes in Bulk GaAs, GaN, and ZnO Semiconductor Microcavities, Phys. Rev. B 78, 235323 (2008).

[48] P. Harrison, F. Long, and W. E. Hagston, Empirical Pseudopotential Calculation of the In-Plane Effective Masses of Electron and Holes of Two-Dimensional Excitons in CdTe Quantum Wells, Superlattices Microstruct. 19, 123 (1996).

[49] D. T. F. Marple and H. Ehrenreich, Dielectric Constant Behavior Near Band Edges in CdTe and Ge, Phys. Rev. Lett. 8, 87 (1962).

[50] Handbook on Physical Properties of Semiconductors, edited by S. Adachi (Kluwer, Boston, 2004), Vol. 3, p. 62.

[51] S. Noda, K. Tomoda, N. Yamamoto, and A. Chutinan, Full Three-Dimensional Photonic Bandgap Crystals at NearInfrared Wavelengths, Science 289, 604 (2000); S. Noda, M. Imada, M. Okano, S. Ogawa, M. Mochizuki, and A. Chutinan, Semiconductor Three-Dimensional and TwoDimensional Photonic Crystals and Devices, IEEE J. Quantum Electron. 38, 726 (2002).

[52] S. Noda, N. Yamamoto, H. Kobayashi, M. Okano, and K. Tomoda, Optical Properties of Three-Dimensional Photonic Crystals Based on III-V Semiconductors at Infrared to Near-Infrared Wavelengths, Appl. Phys. Lett. 75, 905 (1999).

[53] Y. Takahashi, H. Hagino, Y. Tanaka, B.-S. Song, T. Asano, and S. Noda, High-Q Nanocavity with a 2-ns Photon Lifetime, Opt. Express 15, 17206 (2007).

[54] M. O’Neill, M. Oestreich, W. W. Rühle, and D. E. Ashenford, Exciton Radiative Decay and Homogeneous Broadening in $\mathrm{CdTe} / \mathrm{Cd}_{0.85} \mathrm{Mn}_{0.15}$ Te Multiple Quantum Wells, Phys. Rev. B 48, 8980 (1993).

[55] R. André, F. Boeuf, D. Heger, L. S. Dang, R. Romestain, J. Bleuse, and M. Müller, Cavity-Polariton Effects in II-VI Microcavities, Acta Phys. Pol. A 96, 511 (1999).

[56] E. Nelson et al., Epitaxial Growth of Three-Dimensionally Architecture Optoelectronic Devices, Nat. Mater. 10, 676 (2011).

[57] T. J. Kim, Y. D. Kim, and J. Kossut, Study on the Dielectric Function of the CdMgTe Alloy by Using Vacuum Ultraviolet Spectroscopic Ellipsometry, J. Korean Phys. Soc. 49, 1156 (2006).

[58] O. Madelung, Semiconductors: Data Handbook, 3rd ed. (Springer, Berlin, 2004).

[59] M. S. Dresselhaus, G. Dresselhaus, and A. Jorio, Applications of Group Theory to the Physics of Solids, 1st ed. (Springer, New York, 2008).

[60] We use the MIT photonic bands package to do the calculation. See http://ab-initio.mit.edu/wiki/index.php/ MIT_Photonic_Bands.

[61] S. John and S. J. Yang, Electromagnetically Induced Exciton Mobility in a Photonic Band Gap, Phys. Rev. Lett. 99, 046801 (2007).

[62] S. J. Yang and S. John, Exciton Dressing and Capture by a Photonic Band Edge, Phys. Rev. B 75, 235332 (2007).

[63] B. Kuhn-Heinrich, W. Ossau, H. Heinke, F. Fischer, T. Litz, A. Waag, and G. Landwehr, Optical Investigation of
Confinement and Strain Effects in CdTe/(CdMg)Te Quantum Wells, Appl. Phys. Lett. 63, 2932 (1993).

[64] R. Spiegel, G. Bacher, K. Herz, A. Forchel, T. Litz, A. Waag, and G. Landwehr, Recombination and Thermal Emission of Excitons in Shallow CdTe/ $\mathrm{Cd}_{1-x} \mathrm{Mg}_{x} \mathrm{Te}$ Quantum Wells, Phys. Rev. B 53, 4544 (1996).

[65] O. Toader, M. Berciu, and S. John, Photonic Band Gaps Based on Tetragonal Lattices of Slanted Pores, Phys. Rev. Lett. 90, 233901 (2003); O. Toader and S. John, SlantedPore Photonic Band-Gap Materials, Phys. Rev. E 71, 036605 (2005).

[66] M. A. Kinch, M. J. Brau, and A. Simmons, Recombination Mechanisms in 8-14 $\mu \mathrm{HgCdTe}$, J. Appl. Phys. 44, 1649 (1973); J. Bajaj, S. H. Shin, J. G. Pasko, and M. Khoshnevisan, Minority Carrier Lifetime in LPE $\mathbf{H g}_{1-x} \mathbf{C d}_{x}$ Te, J. Vac. Sci. Technol. A 1, 1749 (1983).

[67] W. Shockley and W. T. Read, Jr., Statistics of the Recombinations of Holes and Electrons, Phys. Rev. 87, 835 (1952); R. N. Hall, Electron-Hole Recombination in Germanium, Phys. Rev. 87, 387 (1952).

[68] T. K. Paraïso, M. Wouters, Y. Léger, F. Morier-Genoud, and B. Deveaud-Pédran, Multistability of a Coherent Spin Ensemble in a Semiconductor Microcavity, Nat. Mater. 9, 655 (2010).

[69] A. Das, P. Bhattacharya, J. Heo, A. Banerjee, and W. Guo, Polariton Bose-Einstein Condensate at Room Temperature in an $\mathrm{Al}(\mathrm{Ga}) \mathrm{N}$ Nanowire-Dielectric Microcavity with a Spatial Potential Trap, Proc. Natl. Acad. Sci. U.S.A. 110, 2735 (2013).

[70] O. Penrose and L. Onsager, Bose-Einstein Condensation and Liquid Helium, Phys. Rev. 104, 576 (1956).

[71] W. Ketterle and N. J. van Druten, Bose-Einstein Condensation of a Finite Number of Particles Trapped in One or Three Dimensions, Phys. Rev. A 54, 656 (1996).

[72] N. D. Mermin and H. Wagner, Absence of Ferromagnetism or Antiferromagnetism in One-or Two-Dimensional Isotropic Heisenberg Models, Phys. Rev. Lett. 17, 1133 (1966).

[73] V. Savona, L. C. Andreani, P. Schwendimann, and A. Quattropani, Quantum Well Excitons in Semiconductor Microcavities: Unified Treatment of Weak and Strong Coupling Regimes, Solid State Commun. 93, 733 (1995).

[74] L. Safonova, R. Brazis, and R. Narkowicz, Complex Dielectric Constant of $\mathrm{Cd}_{0.8} \mathrm{Mn}_{0.2}$ Te Crystals Near the Fundamental Absorption Edge, Lith. J. Phys. 44, 421 (2004).

[75] L. S. Dang, G. Neu, and R. Romestain, Optical Detection of Cyclotron Resonance of Electron and Holes in CdTe, Solid State Commun. 44, 1187 (1982).

[76] S. G. Choi, Y. D. Kim, S. D. Yoo, D. E. Aspnes, I. Miotkowski, and A. K. Ramdas, Ellipsometric Studies $\mathrm{Cd}_{1-x} \mathrm{Mg}_{x} \mathrm{Te}(0 \leq x \leq 0.5)$ Alloys, Appl. Phys. Lett. 71, 249 (1997).

[77] J.-H. Yang, S. Chen, W.-J. Yin, X. G. Gong, A. Walsh, and S.-H. Wei, Electronic Structure and Phase Stability of MgTe, ZnTe, CdTe, and Their Alloys in the B3, B4, and B8 Structures, Phys. Rev. B 79, 245202 (2009).

[78] A. A. Kiselev, E. L. Ivchenko, A. A. Sirenko, T. Ruf, M. Cardona, D. R. Yakovlev, W. Ossau, A. Waag, and G. Landwehr, Electron and Hole g Factor Anisotropy in CdTel CdMgTe Quantum Wells, J. Cryst. Growth 184-185, 831 (1998). 